\title{
Dynamic Lifecycle Strategies for Target Date Retirement Funds
}

\author{
Anup Basu \\ Queensland University of Technology
}

\section{Alistair Byrne}

University of Edinburgh

\section{Michael E. Drew*}

Griffith University

\begin{abstract}
Lifecycle funds offered to retirement plan participants gradually reduce their exposure to stocks as they approach the target date of retirement. We show that such deterministic switching rules produce inferior wealth outcomes for the investor compared to strategies that dynamically alter the allocation between growth and conservative assets based on cumulative portfolio performance relative to a set target. The dynamic allocation strategies proposed in this paper exhibit almost stochastic dominance (ASD) over strategies that switch assets unidirectionally without consideration of portfolio performance.
\end{abstract}

\footnotetext{
* Corresponding author: Michael Drew, Professor of Finance, Griffith Business School, Griffith University, Nathan Campus,
} Brisbane, Australia, 4111. Tel: +61 7373 55311; Email: michael.drew@griffith.edu.au 


\section{Introduction}

Target date retirement funds have gained favour with retirement plan investors in recent years.

Typically, these funds initially have a high allocation to stocks but move towards less volatile assets like bonds and cash as the target retirement date approaches. Thus, we are told, they offer the best of both worlds - robust portfolio growth in the early years and preservation of the accumulated wealth as the investor comes closer to retirement. And the best part of all is that once enrolled there is no need for the investors to keep constant watch over their investment strategy. Ameriks and Zeldes (2004) and others have highlighted the problem of inertia among retirement plan participants, which is often manifested in the reluctance to change allocation of their plan assets through time. Since target retirement funds switch assets automatically following a preset glide path laid down by the plan provider, they are thought to be an effective antidote to this apparent flaw in investor behavior.

But does the strategy of switching out of equities with time, popularly known as lifecycle investing, benefit investors? Empirical research has generally found that a switch to low-risk assets prior to retirement can reduce the risk of confronting the most extreme negative outcomes. Lifecycle investment strategies are also said to reduce the volatility of wealth outcomes making them desirable to investors who seek a reliable estimate of final pension a few years before retirement (for example, see Blake, Cairns, and Dowd, 2001). On the other hand, most researchers note that these benefits come at a substantial cost to the investor - giving up significant upside potential of wealth accumulation offered by more aggressive strategies (Booth and Yakoubov, 2004; Byrne et al., 2007). Bodie and Treussard (2007) argue that deterministic target date funds - as commonly implemented are optimal for some investors, but not for others, with suitability depending on the investor's risk aversion and human capital risk.

Our paper questions the rationale for the deterministic nature of lifecycle switching as is the prevalent practice among target retirement funds. We argue that a dynamic switching strategy, which takes into consideration achieved investment returns, will produce superior returns for most investors. The most common argument cited by proponents of deterministic lifecycle switching is apparently straightforward - the probability that returns from stocks will outperform (underperform) those from bonds and cash increases (decreases) with the length of the investment horizon. If this is true, then long horizon investors may prefer to have a higher allocation to stocks in their portfolio compared to investors with shorter investment horizons. $1 \mathrm{It}$ is also argued that younger investors in retirement plans should heavily invest in stocks because they have enough time to recover from a stock market downturn should that happen and can work more to make up for financial losses. On the other hand, for older investors with a few years to retirement, holding such an aggressive portfolio can spell disaster. A major slump in the stock market just before retirement can potentially wipe away years of investment gains with little time to salvage the situation. But would this imply that investors 
should automatically reduce the proportion of stocks in their retirement portfolio as years go by? The following example would explain why the answer is not always yes.

Suppose an investor has a horizon of 40 years. Following popular lifecycle strategies, she decides to put most of her money in stocks for the initial 20 years and then gradually switch to bonds and cash over the last 20 years. Once this allocation decision is made, she puts it on an autopilot (like most lifecycle funds) and goes off to sleep. However, the stock market returns following the investment decision do not augur well for the investor. Due to a prolonged bear market there are several years of negative returns eroding the value of her portfolio. After 20 years, the balance in her account is next to nothing and this gets gradually switched to bonds and cash. Subsequent returns in the account are stable but low. When our Rip Van Winkle investor wakes up after 40 years, she finds herself in a financial situation quite different from what was anticipated while setting the investment strategy. She may even find herself poorer in real terms than what she was 40 years ago.

Undoubtedly the above example is an extreme one and describes only one of the several possibilities that an investor can expect to encounter over a long horizon. Yet it reveals the Achilles' heel of the lifecycle funds currently in market. These funds follow a pre-determined 'Rip Van Winkle' asset allocation strategy where not only the switching of assets is always unidirectional - from stocks to fixed income - but it is also done in proportions that are pre-specified at the inception of the fund. In our example, had the stock market offered very high returns during the last 20 years, the investor would stand to gain very little because her investments were automatically switched from stocks to bonds and cash during that period following the allocation strategy she had set on autopilot. The preprogrammed lifecycle strategy was blind to the fact that she had accumulated too little wealth in the initial years to necessitate switching to conservative assets. The asset switching in that case virtually ensures that she misses the only realistic chance she had to reverse her bad fortune.

The problem for the retirement plan members enrolled in target retirement funds goes even deeper than our hapless investor. Typically the plan members make regular contributions to the retirement account as opposed to a single investment made at the beginning of the 40 year period in our example. As contributions are normally a fixed percentage of the members' salaries, they are expected to grow larger over time with growth in earnings. Therefore, as Shiller (2005) points out, the lifecycle strategy is heavily in the stock market in the early years when the contribution size is relatively small and switches out of it when earnings and contributions grow larger in later years. This can be counterproductive as by moving away from stocks to low return assets just when the size of their contributions (and accumulated fund) are growing larger, the investor may be foregoing the opportunity to earn higher returns on a larger sum of money invested. Basu and Drew (2008) confirm this view by demonstrating that the growth in portfolio size with time is important from an asset allocation perspective and by ignoring this phenomenon, lifecycle strategies tend to dampen the growth potential of the retirement investor's portfolio in most cases. 
One cannot help wondering why lifecycle funds need to have their benchmark asset allocation policy cast in stone. A possible alternative approach suggested by Basu and Drew (2008) is to switch to conservative assets a few years before retirement only if the accumulation in the retirement account meets or exceeds the plan member's accumulation target at that stage thereby facilitating a move to lock in some of the gains. By the same token, the plan member may decide not to switch to less volatile (but low return) assets where the past performance of the portfolio has been unsatisfactory, leaving him or her with inadequate wealth relative to the target. In this case, the decision to switch or not to switch and even how much to switch at any stage depend on the cumulative performance of the retirement portfolio in the preceding years.

In this paper, we extend this alternative approach by proposing a dynamic lifecycle strategy which is flexible in adjusting its allocation between growth and conservative assets as the retirement date approaches depending on the extent that the plan member's wealth accumulation objective has been achieved at that time. In other words, this strategy is responsive to past performance of the portfolio relative to the investor's target return in determining the right mix of assets in future periods. ${ }^{2}$ While initially it invests heavily in equities just as any other lifecycle strategy, the switching to fixed income is not automatic. It only takes place if the investor has accumulated wealth in excess of the target accumulation at the point of switch. Also, after switching to conservative assets, if the accumulation falls below the target in any period, the direction of switch is reversed by moving away from fixed income and towards stocks. We compare and contrast the outcomes of this dynamic strategy with those achieved by following a regular deterministic lifecycle strategy. ${ }^{3}$

\section{Comparing Deterministic and Dynamic Lifecycle Strategies}

In comparing conventional deterministic lifecycle and dynamic lifecycle strategies, we consider the case of a hypothetical individual who joins the plan with starting salary of $\$ 25,000$. The earnings grow linearly at the rate of $4 \%$ per year over the next 41 years, the duration of the individual's working life. Throughout this period, regular annual contributions amounting to $9 \%$ of earnings go into the retirement plan account. ${ }^{4}$ We assume that the contributions are credited annually to the member's account at the end of each year. This means that the first contribution by the member is made at the end of the first year followed by 39 more contributions in as many years. No contribution is made in the final year of employment.

Our hypothetical plan member can choose between a conventional lifecycle strategy and a dynamic strategy. We consider two variations of the conventional lifecycle strategy, namely $L C_{20,20}$ and $L C_{30,10}$, which invest in a $100 \%$ stocks portfolio for 20 years and 30 years respectively following the first contribution. Thereafter both of them switch linearly from stocks to bonds and cash over the remaining 20 (or 10) years in such a manner that at the point of retirement all assets are held in bonds and cash. This type of allocation is typical of lifecycle or target date strategies used in practice. 
Similarly, the dynamic strategy has two variations, namely $D L C_{20,20}$ and $D L C_{30,10}$. They invest in the same $100 \%$ stocks portfolio as the two lifecycle strategies during the first 20 (and 30) years. Thereafter each year the strategies review how the portfolio has performed relative to the investor's accumulation objective. If the value of the portfolio at any point is found to equal or exceed the investor's target, the portfolio partially switches to conservative assets. Otherwise, it remains invested $100 \%$ in stocks. If the switch to conservative assets has begun and the cumulative performance drops below target, the fund is switched back into growth assets. From our formulation of the strategies, it is clear that while $D L C_{20,20}$ and $D L C_{30,10}$ uses performance feedback control in switching assets, $L C_{20,20}$ and $L C_{30,10}$ do not.

Although individuals may have different accumulation objectives on retirement, we need to make an assumption about the accumulation target set by the hypothetical individual employing the dynamic allocation strategies in this paper. Dimson, Marsh, and Staunton (2002) have compiled returns for US stocks, bonds, and bills from 1900. We use an updated version of their dataset and find the geometric mean return offered by US stocks between 1900 and 2004 is $9.69 \%$. We assume that the individual sets a target of achieving a return close to this rate, say $10 \%$, on the retirement plan investments. In other words, the retirement portfolio under the dynamic strategy aims to closely match the compounded accumulation of a fund where contributions are annually reinvested at $10 \%$ nominal rate of return.

For $D L C_{20,20}$ which invests in $100 \%$ stocks portfolio for 20 years, we assume that the individual sets a target of $10 \%$ compounded annual rate of return on investment for the initial 20 year period. At the end of 20 years, if the actual accumulation in the retirement account exceeds the accumulation target, the assets are switched to a relatively conservative portfolio comprising of $80 \%$ stocks and $20 \%$ fixed income (equally split between bonds and cash). However, if the actual accumulation in the account is found to fall below the target, the portfolio remains invested in $100 \%$ stocks. This performance review process is carried out annually for the next 10 years and the asset allocation is adjusted depending on whether the holding period return is greater or less than the target, which remains set at a $10 \%$ annualized return on a cumulative basis. In the final 10 years the same allocation principle is applied with one difference. If the value of the portfolio in any year during this period matches or exceeds the investor's target accumulation (i.e. $10 \%$ annualized cumulative return) at that point, $60 \%$ of assets are invested in equities and $40 \%$ in fixed income (equally split between bonds and cash). Failing to achieve the target return for the holding period, results in all assets being invested in the $100 \%$ stocks portfolio.

Similar principles are applied for $D L C_{30,10}$, which invests in $100 \%$ stocks for the 30 years after making the first contribution. After 31 years, if the portfolio value in any year matches or exceeds the 
target accumulation, $20 \%$ assets are switched to fixed income (equally split between bonds and cash). A failure to achieve the target performance results in the portfolio being invested in $100 \%$ equities. The performance of the portfolio relative to the target is monitored annually and the asset allocation is adjusted accordingly. In the final 5 years before retirement, if the portfolio performance at any point matches or exceeds the target accumulation at that point, $40 \%$ of assets are switched to fixed income (equally split between bonds and cash).

\section{Simulating Wealth Outcomes}

To generate simulated investment returns under the two conventional lifecycle strategies (say

$L C_{20,20}$ and $L C_{30,10}$ ) and their corresponding dynamic lifecycle strategies $\left(D L C_{20,20}\right.$ and

$D L C_{30,10}$ ) we use an updated version of the dataset of annual nominal returns for US stocks, bonds, and bills originally compiled by Dimson, Marsh, and Staunton (2002) and commercially available through Ibbotson Associates. The descriptive statistics are presented in Table 1. The dataset spans a period of 105 years between 1900 and 2004 and thus captures both favorable and unfavorable returns on the individual asset classes over the entire twentieth century. However, to examine holding period returns of assets over horizons as long as 40 years, 105 years worth of returns data may not be sufficient. There are only two independent, non-overlapping 40-year holding period observations within our dataset. Any conclusion based on a sample of two observations cannot be deemed reliable.

\section{Table 1. Descriptive Statistics of Nominal Returns Data}

\begin{tabular}{llll}
\hline & Stocks & Bonds & Cash \\
\hline Mean & & & \\
Median & $11.6 \%$ & $5.3 \%$ & $4.1 \%$ \\
Maximum & $14.0 \%$ & $4.0 \%$ & $4.0 \%$ \\
Minimum & $58.0 \%$ & $40.0 \%$ & $15.0 \%$ \\
Standard Deviation & $-44.0 \%$ & $-9.0 \%$ & $0.0 \%$ \\
Skewness & $20.0 \%$ & $8.2 \%$ & $2.9 \%$ \\
Kurtosis & -0.32 & 1.53 & 0.72 \\
Observations & 2.78 & 6.68 & 4.18 \\
& 105 & 105 & 105 \\
\hline
\end{tabular}

To get around the problem of insufficient data, we use bootstrap resampling. The empirical annual return vectors for the three asset classes in the dataset is randomly resampled with replacement to generate asset class return vectors for each year of the 40 year investment horizon confronting the two hypothetical retirement plan investors. Since we randomly draw rows (representing years) from the matrix of asset class returns, we are able to retain the cross-correlation between the asset class returns as given by the historical data series while assuming that returns for individual asset classes are independently distributed over time. 
As the resampling is done with replacement, a particular data point from the original data set can appear multiple times in a given bootstrap sample. This is particularly important while examining probability distribution of future outcomes. For example, 1931 is the worst year for stock market in our 105 year long dataset. In that year return from stocks was $-44 \%$ while bonds and bills offered returns of $1 \%$ and $-5 \%$ respectively. Although this is only one observation in the century long data, a bootstrap sample of 40 yearly returns can include this return observation for 1931 many times in any sequence. Similarly, return observations for other years, good or bad, can also be repeated a number of times within a bootstrap sample. Since this method allows for inclusion of such extreme possibilities (like a $-44 \%$ return occurring a number of times in a particular 40 -year long return path), by obtaining a large number of bootstrap samples from the observed historical data, one can capture a much wider range of future possibilities.

The asset class return vectors obtained by bootstrap resampling are combined with their respective weightings under each asset allocation strategy to generate portfolio returns for each year in the 40 year horizon. The simulation trial is iterated 10,000 times for lifecycle strategy $L C_{20,20}$ and its corresponding dynamic strategy $D L C_{20,20}$ thereby generating 10,000 independent 40 year return paths that would govern the possible wealth outcomes for the individuals following them.. A separate set of experiment (comprising of another 10,000 trials) is conducted for the other pair of lifecycle and dynamic strategies, $L C_{30,10}$ and $D L C_{30,10}$. For the purpose of doing a comparative analysis, we include two other allocation strategies - (i) a 100\% stocks strategy and (ii) a balanced strategy which allocates in the ratio of 60:30:10 between stocks, bonds, and cash - in both sets of experiments and provide the results in next section.

\section{Simulation Results}

The resampling method described above generates a range of terminal wealth outcomes under the conventional lifecycle strategies and their corresponding dynamic strategies. The parameter estimates for the wealth distribution under the different strategies are reported in Table 2. From panel $A$, which provides the results for the conventional lifecycle and dynamic lifecycle strategies that remain invested in $100 \%$ stocks for the first 20 years, the difference is stark. The mean and the median outcome for the dynamic lifecycle strategy $D L C_{20,20}$ exceeds those for the conventional lifecycle strategy $L C_{20,20}$ by more than half a million dollars! The first quartile and the third quartile estimate for the former are also greater than the latter by $\$ 245,033$ and $\$ 704,324$ respectively. For the lifecycle strategies which always invest in the $100 \%$ stocks portfolio for the first 30 years, the results appear in Panel B. As in panel $\mathrm{A}$, we find that the dynamic lifecycle strategy $D L C_{30,10}$ produces much higher mean, median, first and third quartile outcomes than the conventional lifecycle 
strategy $L C_{30,10}$. The gap between the outcomes in this case, however, is lower than what it was between $D L C_{20,20}$ and $L C_{20,20}$. This is expected as $D L C_{30,10}$ and $L C_{30,10}$ strategies invest in the same portfolio (100\% stocks) for ten more years.

\section{Table 2. Terminal Value of Retirement Portfolio in Nominal Dollars}

\begin{tabular}{|c|c|c|c|c|}
\hline Strategy & Mean & Median & $25^{\text {th }}$ Percentile & $75^{\text {th }}$ Percentile \\
\hline \multicolumn{5}{|l|}{ Panel A } \\
\hline$D L C_{20,20}$ & $1,978,387$ & $1,733,256$ & $1,037,838$ & $2,432,030$ \\
\hline$L C_{20,20}$ & $1,426,510$ & $1,163,836$ & 792,805 & $1,727,706$ \\
\hline $100 \%$ Stock & $2,523,681$ & $1,715,014$ & 981,005 & $3,040,650$ \\
\hline Balanced & $1,273,744$ & $1,117,258$ & 804,466 & $1,562,407$ \\
\hline \multicolumn{5}{|l|}{ Panel B } \\
\hline Dynamic & $2,243,825$ & $1,762,712$ & 988,573 & $2,695,902$ \\
\hline Lifecycle & $1,919,124$ & $1,408,545$ & 876,404 & $2,340,550$ \\
\hline $100 \%$ Stock & $2,547,867$ & $1,716,608$ & 965,411 & $3,102,896$ \\
\hline Balanced & $1,276,875$ & $1,118,547$ & 799,502 & $1,573,030$ \\
\hline
\end{tabular}

Note: Results are based on 10,000 simulations.

In addition to the conventional and the dynamic lifecycle strategies, which are of primary interest in this paper, we also simulate for comparison wealth outcomes for the $100 \%$ stocks and the balanced strategy. The mean outcomes for the $100 \%$ stocks strategy are higher than both the conventional and dynamic strategy pairs. Given the existence of a large positive equity premium in our data, this is unsurprising. While the median and the first quartile outcomes for the $100 \%$ stocks strategy is higher than those of $L C_{20,20}$ and $L C_{30,10}$ they fall short of both $D L C_{20,20}$ and $D L C_{30,10}$. This suggests that dynamic strategies are superior in protecting investors from the risk of adverse outcomes than both the aggressive $100 \%$ stocks strategy and the conventional lifecycle strategy, which adopts a predetermined conservative allocation in later years.

The ineffectiveness of lifecycle switching in protecting investors from the risk of confronting adverse wealth outcomes on retirement is clear when we look at the balanced fund simulation results. The balanced fund, whose mean and median outcomes are inferior to all the other three strategies, outperforms $L C_{20,20}$ in terms of the first quartile estimate. This appears to put a question mark on the efficacy of the conventional lifecycle strategies. Dynamic lifecycle strategies, again, seem to produce better results in this respect. But we take up this issue later in the paper. 
Despite the dynamic strategies ( $D L C_{20,20}$ and $D L C_{30,10}$ ) outperforming their conventional lifecycle counterparts ( $L C_{20,20}$ and $L C_{30,10}$ ) in terms of the mean, median, and the lower and upper quartile outcomes, can we conclude they are superior investment vehicles for the retirement plan members? This cannot be answered with certainty without comparing the entire range of outcomes under the two approaches. Stochastic Dominance is a well known approach used in this type of situation because it relies on the entire distribution of outcomes. ${ }^{5}$ It also places minimal restrictions on the investors' utility functions and makes no assumptions (such as normality) about the distributions. Stochastic Dominance approach has been employed in a wide range of areas including investments, operations research, medicine, and agriculture. ${ }^{6}$ We use this approach here to find out whether investors would prefer the terminal wealth distribution under one asset allocation strategy over that of the other.

Formally, given utility of wealth is a non-decreasing function i.e. $U^{\prime}(W) \geq 0$, if $F$ and $G$ represents respectively the cumulative distributions of terminal wealth outcomes under the dynamic lifecycle strategy and the conventional lifecycle strategy, the former dominates the latter under the stochastic dominance (SD) rule if and only if:

$$
F(W) \leq G(W) \quad \forall W
$$

In plain words, this means that the dynamic lifecycle strategy would dominate the corresponding conventional lifecycle strategy by the SD criterion if the cumulative distribution of terminal wealth outcomes under it always remains below the cumulative wealth distribution of the conventional lifecycle strategy. This rule is also known as First Degree Stochastic Dominance (FSD). ${ }^{7}$

One serious limitation of Stochastic Dominance approach in ranking alternatives is that it operates under very restrictive condition often violated in real world situations. ${ }^{8}$ In view of this difficulty, an alternative has been proposed in the literature in the form of Almost Stochastic Dominance (ASD) (Leshno and Levy, 2002) which captures all reasonable preferences and therefore, is acceptable as an ordering criterion by most decision makers. ASD allows for violation of the condition that $F$ has to always remain below $G$ for the former to dominate the later as long as the area between $F$ and $G$ which causes the violation (left of point $\mathrm{X}$ ) is very small compared to the total area between the two distributions. If $\varepsilon$ denotes the ratio between the area of FSD violation and the total area between $F$ and $G$, then smaller $\varepsilon$ is, smaller is the area of violation relative to the full range of outcomes and more investors would prefer $F$ over $G$. In other words, $F$ is said to have 'Almost FSD dominance' over G. Although the magnitude of $\varepsilon$ presumably is different for different set of investors, an experimental study conducted among undergraduate and graduate students and mutual fund managers by Levy, Leshno, and Leibovitch (2006) estimates the value of $\varepsilon$ to be $5.9 \%$ or 0.059 . To apply the ASD rule 
extremely conservatively, we would consider $0<\varepsilon<0.01$ as acceptable for dominance by ASD, where there is no clear dominance by FSD. Setting such a low threshold of 0.01 for $\varepsilon$ would eliminate any realistic chance of error on our part in applying the ASD criterion.

Figure 1 demonstrates the cumulative distributions of terminal wealth achieved under $L C_{20,20}$ and $D L C_{20,20}$ strategies. Again for the purpose of comparison, we also show cumulative wealth distributions for the $100 \%$ stocks and the balanced strategies. The horizontal axis of the graph represents the nominal dollar value of the portfolio at the point of retirement. As explained above, if the CDF for one strategy lies under (or to the right of) other CDFs, it is likely to result in a superior outcome relative to other strategies. Also, if CDF for a strategy is generally steeper than the others, the strategy can be considered to result in less variable outcomes.

\section{Figure 1. Cumulative Distribution Plots for the First Pair of Lifecycle and Dynamic Strategies $\left(L C_{20,20}\right.$ and $\left.D L C_{20,20}\right)$}

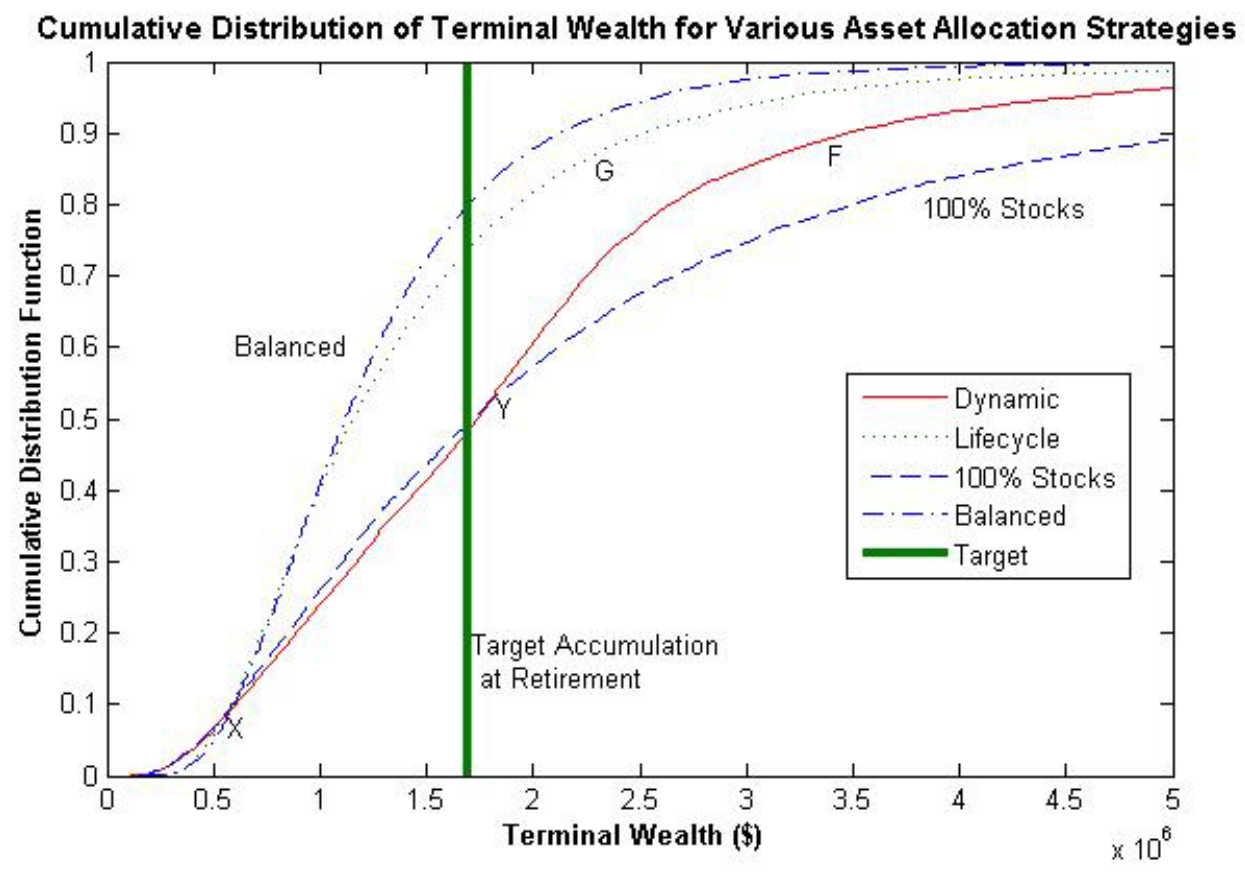

It is clear that except for a very small part on the left of the point $X$, the cumulative distribution plot of $D L C_{20,20}$ remains much under that of $L C_{20,20}$. Therefore the dynamic lifecycle strategy dominates the conventional lifecycle strategy on the right of point $X$ but not on the left of it. Therefore there is violation of the strict SD criterion, the area of violation being denoted by the area between the distribution plots $F$ and $G$ to the left of $X$. 
Except for a very small section to the left of point $X$ representing wealth outcomes of about $\$ 500,000$ or less after 41 years, we can infer from the cumulative distributions that the investor employing $D L C_{20,20}$ has higher chance of achieving any particular accumulation outcome than the investor employing $L C_{20,20}$. For example, the former has about $75 \%$ probability of accumulating more than one million dollars at retirement whereas the later has got only a $60 \%$ chance of crossing that milestone. If investors set a target of achieving a compounded return of $9 \%$ minimum on their investments, which amounts to an accumulated wealth of at least $\$ 1.69$ million at retirement, our results indicate that the $D L C_{20,20}$ strategy would achieve this goal with almost $50 \%$ certainty. With $L C_{20,20}$ strategy, this probability drops to only $25 \%$. The gap between the cumulative distribution functions for the two strategies widens as we move up further towards higher accumulation figures although after a point (roughly around two million dollars) its starts diminishing gradually.

A comparison of the cumulative distributions of the lifecycle strategies $L C_{20,20}$ and $D L C_{20,20}$ with that of the $100 \%$ stock strategy reveals two important results. First, we find that the distribution of the conventional lifecycle strategy $L C_{20,20}$ always remains above that of the $100 \%$ stocks strategy except for the small section to the left of point X (representing only about the worst $5 \%$ of outcomes). This questions the effectiveness of conventional lifecycle strategies in protecting investors' wealth from the vagaries of stock market downturns. Had it been the case, we would have found $X$ much to the right of its current location i.e. $L C_{20,20}$ would have dominated the $100 \%$ stock strategy for a much larger percentage of outcomes in the lower end of the distribution. In contrast, we find the cumulative distribution of $D L C_{20,20}$ remains below that of the $100 \%$ stock strategy for a much longer section (the left side of $\mathrm{Y}$ ). This clearly suggests its effectiveness in reducing the risk of investor's wealth breaching any floor level of wealth to the left of Y. It does much better in terms of producing superior outcomes in the below median range, which is likely to be viewed as the zone of risk for most investors. Remarkably it is obvious from the diagram that our hypothetical investor has a slightly higher chance of achieving the target wealth outcome of \$1.69 million by employing the $D L C_{20,20}$ instead of the $100 \%$ stocks strategy.

Now we turn our attention to Figure 2 which shows the cumulative wealth distribution functions for the other lifecycle and dynamic strategy pair - $L C_{30,10}$ and $D L C_{30,10}$. As before, we also show cumulative wealth distributions for the $100 \%$ stocks and the balanced strategies. Apart from a small part in the extreme lower tail of the distributions representing terminal wealth outcomes below $\$ 500,000$, the cumulative wealth distribution function of $D L C_{30,10}(\mathrm{~F})$ always remains below that of $L C_{30,10}(\mathrm{G})$. As is the case with $L C_{20,20}$ and $D L C_{20,20}$ pair, the distance between the CDF plots is 
larger in the middle than in the extremes. In other words, the dynamic strategy dominates the conventional strategy over a range of outcomes by a wide margin.

\section{Figure 2. Cumulative Distribution Plots for the Second Pair of}

Lifecycle and Dynamic Strategies $\left(L C_{30,10}\right.$ and $\left.D L C_{30,10}\right)$

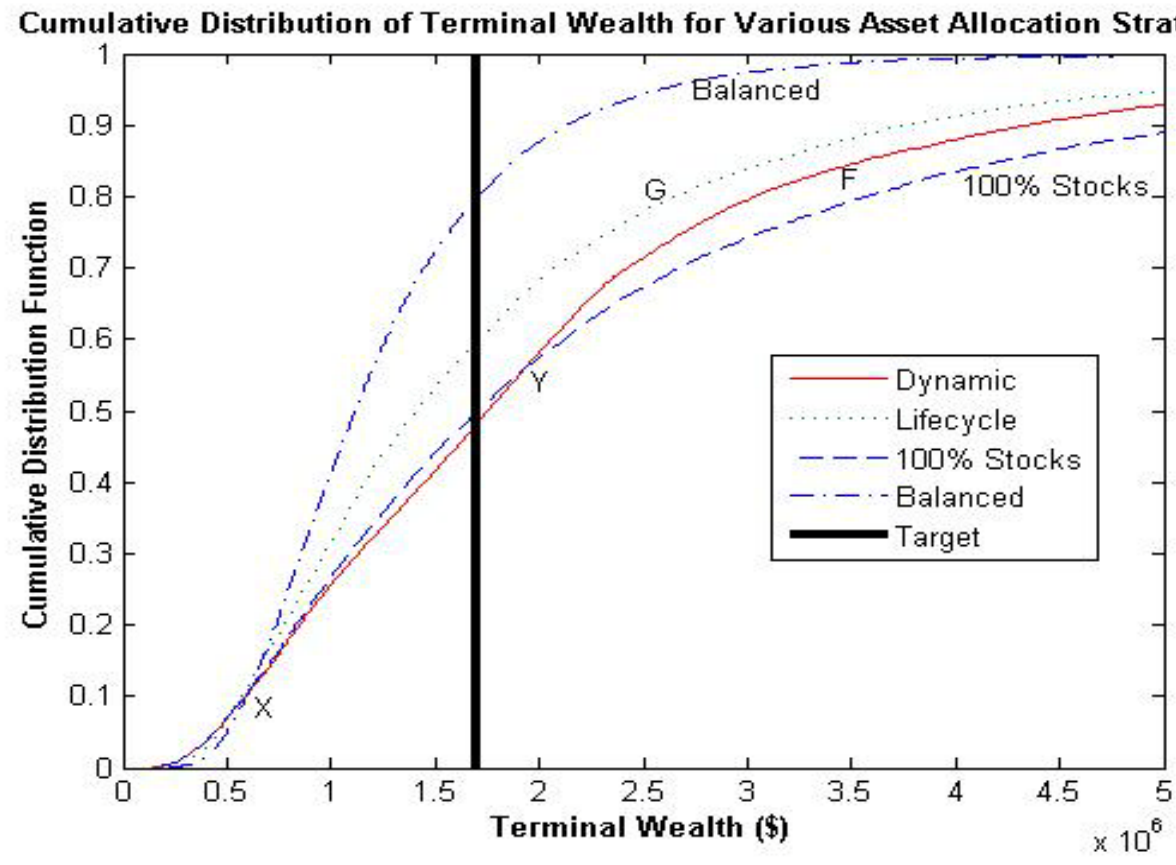

In relation to the target accumulation outcome of $\$ 1.69$ million at retirement, Figure 2 indicates that the $L C_{30,10}$ strategy would achieve this goal with about $40 \%$ certainty. Although this is significant improvement compared to the performance of $L C_{20,20}$, it still falls short of the corresponding dynamic strategy, $D L C_{30,10}$, which surpasses the target on more than $50 \%$ of occasions. The cause for $L C_{30,10}$ putting up a superior performance relative to $L C_{20,20}$ strategy in attaining the target may be attributed mainly to the fact that the former invests in a $100 \%$ stocks portfolio for a longer duration (30 years) compared to that of the latter (20 years). However, to apply the same argument to explain the dominance of dynamic strategies over corresponding lifecycle strategies appears too simplistic. Had this been the only reason, the $100 \%$ stocks strategy would have outperformed other strategies in terms of exceeding the target accumulation. But as is evident from Figure 2, the probability of achieving the target wealth outcome with $D L C_{30,10}$ strategy is clearly higher than that 
with $100 \%$ stocks strategy. Also, the median outcome for $D L C_{30,10}$ strategy is larger than that of $100 \%$ stock strategy.

\section{Table 3. Almost Stochastic Dominance Results for Dynamic strategies}

Strategy Area of SD Violation Relative to Non-violation $(\varepsilon)$

\begin{tabular}{|c|c|c|c|c|}
\hline \multicolumn{5}{|c|}{ Panel A } \\
\hline & Lifecycle ( $L C_{20,20}$ ) & Balanced & $D L C_{20,20}$ & $100 \%$ Stocks \\
\hline \multicolumn{5}{|l|}{ Dynamic } \\
\hline$\left(D L C_{20,20}\right)$ & $0.0067^{*}$ & $0.0068^{*}$ & - & 0.9624 \\
\hline \multirow[t]{3}{*}{$100 \%$ Stocks } & $0.0039^{*}$ & $0.0046 *$ & 0.0375 & - \\
\hline & & $\underline{\text { Panel B }}$ & & \\
\hline & Lifecycle ( $L C_{30,10}$ ) & Balanced & $D L C_{30,10}$ & $100 \%$ Stocks \\
\hline \multicolumn{5}{|l|}{ Dynamic } \\
\hline$\left(D L C_{30,10}\right)$ & $0.0072^{*}$ & $0.0058^{*}$ & - & 0.9424 \\
\hline $100 \%$ Stocks & $0.0092 *$ & $0.0046^{*}$ & 0.0575 & - \\
\hline
\end{tabular}

* Almost Stochastic Dominance exists for the threshold value $0<\varepsilon<1$

To find out whether dominance by ASD exists between different strategies, we calculate the values of $\varepsilon$ and provide the results in Table 3 . There is strong evidence to suggest that $D L C_{20,20}$ dominates $L C_{20,20}$ under ASD since $\varepsilon$ is .0067 which is far less than our threshold area of violation of $1 \%$. Similarly $D L C_{20,20}$ dominates the balanced strategy with the value of $\varepsilon$ being 0.0068 . However it does not dominate the $100 \%$ stock strategy under ASD criterion. The $100 \%$ stocks strategy also clearly dominates the lifecycle and balanced strategy with even lower values of $\varepsilon$ in both cases. There is no ASD over the dynamic strategy at our set threshold $\varepsilon$ of $1 \%$. This result would change if the threshold is set somewhat higher, say at $5 \%$ as the $100 \%$ stocks strategy produces some spectacularly high simulation outcomes which increase the area of non-violation thereby reducing the value of $\varepsilon$ when measuring its dominance over others.

For dynamic and lifecycle strategy pairs commencing the switch after 30 years, the results are shown in panel B. For $D L C_{30,10}$ and $L C_{30,10}$, the evidence for ASD in favour of the former is even stronger than that in the other pair with $\varepsilon$ of 0.0072 . Similarly, the dominance over the balanced portfolio is also slightly stronger, the $\varepsilon$ value in this case being 0.0058 . As expected, the $100 \%$ stocks strategy also dominates both the lifecycle and the balanced strategies. Comparing $D L C_{30,10}$ and the $100 \%$ stocks 
strategy, neither of the strategies dominates the other although the values of $\varepsilon$ indicate that the $100 \%$ stocks strategy comes close to having ASD over the dynamic strategy. Again, this is clearly a result of $100 \%$ stocks strategy beating the other strategies by wider margins as we move towards the right end of the distribution.

But what is the success (or failure) rate of the dynamic strategy over other strategies in different possible future states of the world? This knowledge is important to the investor yet comparing probability distributions of terminal wealth under different competing strategies does not provide a clear answer. This is because in doing so we are comparing the $n$-th percentile outcome of one strategy with the $n$-th percentile outcome of the other. In plain words, the good scenarios under one strategy are compared to the good scenarios under another and likewise the bad outcomes are pitted against the bad outcomes. But for any particular future state of the world (with a particular asset return path over the investment horizon), this comparison may not be very useful. For example, if stock returns turn out to be very poor compared to other assets in a particular state of the world, the $100 \%$ stocks strategy would produce inferior outcome relative to a balanced strategy no matter how attractive or dominating the wealth distribution of the former appears compared to the latter.

Recall that the asset class return path over the 41 year horizon is unique for each trial in our simulation experiment. Each of those 10,000 trials represents a different possible future state of the world. Therefore, for each trial, we compare the wealth outcomes under all four strategies, the main point of interest being how the dynamic strategy perform vis-à-vis other strategies. To be specific, we compute the shortfall probability as of $D L C_{20,20}$ and $D L C_{30,10}$ as well their average size of shortfall compared to the other three strategies. These shortfall measures are likely to constitute an important part of what the investors view as the downside risk of adopting the dynamic allocation strategy. The results, provided in Table 4, show that the dynamic strategy has a small chance of underperforming the conventional lifecycle strategy. The wealth outcome of the dynamic strategy $D L C_{20,20}$ falls short of that of the corresponding lifecycle strategy $L C_{20,20}$ in only $19 \%$ of trials. For $D L C_{30,10}$, the chance of it underperforming the corresponding lifecycle strategy $L C_{30,10}$ however increases to $26 \%$ i.e. one-in-four. However, the average size of the shortfall in both the cases is small $(\$ 34,462$ and $\$ 50,273)$ compared to the average size of terminal wealth outcomes, which run into millions.

Table 4. Shortfall Measures of Dynamic Strategies Relative to other Asset Allocation Strategies

\begin{tabular}{lll}
\hline Strategy & Shortfall Probability & Average Shortfall (\$) \\
\hline$D L C_{20,20}$ & & \\
& $19 \%$ & 34,462
\end{tabular}




\begin{tabular}{|c|c|c|}
\hline Lifecycle ( $L C_{20,20}$ ) & & \\
\hline $100 \%$ Stocks & $51 \%$ & 582,815 \\
\hline Balanced & $10 \%$ & 6,110 \\
\hline$D L C_{30,10}$ & & \\
\hline Lifecycle ( $L C_{30,10}$ ) & $26 \%$ & 50,273 \\
\hline $100 \%$ Stocks & $43 \%$ & 343,890 \\
\hline Balanced & $11 \%$ & 6,907 \\
\hline
\end{tabular}

Note: Results are based on 10,000 simulations.

[Table 4 about here]

Further comparing individual trial outcomes, we find that the $D L C_{20,20}$ strategy gives the $100 \%$ stock strategy a close run. The chance of doing better with either strategy is almost even with the $100 \%$ stocks strategy emerging the winner in $51 \%$ of the trials. But when compared with $D L C_{30,10}$ ， the $100 \%$ stock strategy fares better only in $43 \%$ of trials i.e. the dynamic strategy emerges winner in a majority of cases. The average size of the shortfall for the dynamic strategy in both cases, however, is quite high at $\$ 582,815$ and $\$ 343,890$ respectively. This is not unexpected with the $100 \%$ stocks strategy producing several spectacularly large wealth outcomes in the above median and particularly in the upper quartile. Relative to the balanced strategy, the chance of underperformance of the dynamic strategy is minimal. The $D L C_{20,20}$ and $D L C_{30,10}$ strategies underperforms the balanced strategy only in $10 \%$ and $11 \%$ of the trials respectively. The average size of shortfall in both cases is extremely small at $\$ 6,110$ and $\$ 6,907$ respectively.

While our evidence so far suggests the superiority of dynamic strategies over conventional lifecycle strategies, the saving grace for the latter may lie in the zone of the most adverse outcomes. This is represented by the left portion of $X$ in the CDF plots in Figures 1 and 2 where the lifecycle strategies actually dominate corresponding dynamic strategies. It is also apparent from the figures that this zone is constituted by outcomes that are below the $10^{\text {th }}$ percentile mark for every strategy. To have some idea about how large the differences are between the adverse outcomes under different strategies, we report the value-at-risk (VaR) estimates at confidence levels of $99 \%, 95 \%$, and $90 \%$ for both sets of simulation trials in Table 5. We also estimate the expected tail loss (ETL) at a 95\% confidence level which is essentially a probability weighted average of all below-VaR outcomes at that specified level of confidence. 


\section{Table 5. VaR and ETL Estimates for Different Asset Allocation Strategies}

\begin{tabular}{|c|c|c|c|c|}
\hline \multirow{2}{*}{ Asset Allocation Strategy } & \multicolumn{3}{|c|}{ VaR at Different Confidence Levels } & \multirow{2}{*}{$\begin{array}{l}\text { ETL at 95\% Confidence } \\
\text { Level }\end{array}$} \\
\hline & $99 \%$ & $95 \%$ & $90 \%$ & \\
\hline \multicolumn{5}{|l|}{ Panel A } \\
\hline Dynamic ( $D L C_{20,20}$ ) & 275,914 & 461,640 & 607,872 & 344,437 \\
\hline Lifecycle ( $L C_{20,20}$ ) & 375,810 & 486,156 & 578,814 & 417,804 \\
\hline $100 \%$ Stocks & 271,458 & 447,330 & 592,348 & 337,980 \\
\hline Balanced & 361,326 & 505,209 & 597,506 & 422,350 \\
\hline \multicolumn{5}{|l|}{$\underline{\text { Panel B }}$} \\
\hline Dynamic ( $D L C_{30,10}$ ) & 274,968 & 444,468 & 599,673 & 340,901 \\
\hline Lifecycle ( $\left.L C_{30,10}\right)$ & 321,875 & 468,598 & 581,526 & 377,114 \\
\hline $100 \%$ Stocks & 274,657 & 443,251 & 595,398 & 339,980 \\
\hline Balanced & 369,362 & 501,541 & 599,863 & 423,124 \\
\hline
\end{tabular}

As is evident in the CDF plots, both the lifecycle strategies $L C_{20,20}$ and $L C_{30,10}$ produce $95 \%$ and 99\% VaR estimates compared to their dynamic counterparts (and 100\% stocks strategy). The differences between the $95 \%$ VaR estimates (less than $\$ 25,000$ ) do not appear to be large enough to ring any alarm bells. But when one compares the $99 \%$ VaR estimates, the differences between the lifecycle and dynamic strategy grow considerably larger. The estimated 99\% VaR estimate for $L C_{20,20}$ strategy is almost $\$ 100,000$ more than that of the corresponding dynamic strategy $D L C_{20,20}$. Between $L C_{30,10}$ and $D L C_{30,10}$, the corresponding difference, however, is smaller than $\$ 50,000$.

Yet one would be reluctant to declare lifecycle funds to be the preferred investment strategy even under the unreasonable assumption that investors care only about the zone of extremely adverse wealth outcomes (below $10^{\text {th }}$ percentile in this case). This is because the balanced fund produces better $95 \%$ VaR estimate than both $L C_{20,20}$ and $L C_{30,10}$. In terms of $99 \%$ VaR estimates, the 
balanced fund outperforms $L C_{30,10}$ but underperforms $L C_{20,20}$. When we consider the average for all outcomes below 95\% VaR estimates, the balanced fund produces ETL estimates that are higher than both $L C_{20,20}$ and $L C_{30,10}$. These results suggest that if the retirement plan investors are concerned about improving the floor level of possible wealth outcomes or protection from extreme downside risk, they would be better off by investing in a static balanced fund rather than a conventional lifecycle fund.

\section{Sensitivity Analysis}

How sensitive are our results to the target return used by the dynamic strategies to switch allocations? Recall that both the dynamic strategies in our study use a switching rule are based on a target return of $10 \%$ on investment. We repeat the simulation trials using target returns in the 8 to $12 \%$ range but do not find any evidence of the dominance of the dynamic strategies over corresponding lifecycle strategies (and balanced strategy) disappearing at all. The results are reported in Tables 6 to 9. An increase in target return leads to a slightly higher chance of the dynamic strategy underperforming the lifecycle strategy. For example, when the target rate of return is set at $12 \%$, the shortfall probability of the dynamic strategy $D L C_{20,20}$ relative to lifecycle strategy $L C_{20,20}$ is $20 \%$. This is just $1 \%$ higher than the shortfall probability with the target return set at $10 \%$. The corresponding increase in average shortfall of $D L C_{20,20}$ is also very small (less than $\left.\$ 5,000\right)$. Similarly, a decrease in target return results in a very small reduction in the shortfall probability of the dynamic strategies. With a target return of $8 \%$, the shortfall probability of dynamic strategy

$D L C_{20,20}$ relative to lifecycle strategy $L C_{20,20}$ is $18 \%$. Again, the average shortfall is slightly above $\$ 5,000$. The estimates of ASD for simulations with these different target returns are remarkably similar to those with $10 \%$ target rate of return and therefore do not alter our conclusions in the previous section. 
Table 6: Terminal Value of Dynamic Portfolio in Nominal Dollars for Different Target Returns

\begin{tabular}{lcccc}
\hline $\begin{array}{l}\text { Target Rate } \\
\text { of Return }\end{array}$ & Mean & Median & 25th Percentile & 75th Percentile \\
\hline Dynamic ( $D L C_{20,20}$ ) & & & & \\
$8 \%$ & $1,909,042$ & $1,517,123$ & $1,054,035$ & $2,281,629$ \\
$9 \%$ & $1,933,004$ & $1,639,719$ & $1,048,390$ & $2,339,969$ \\
$10 \%$ & $1,978,387$ & $1,733,256$ & $1,037,838$ & $2,432,030$ \\
$11 \%$ & $2,051,114$ & $1,769,992$ & 991,135 & $2,656,386$ \\
$12 \%$ & $2,120,623$ & $1,772,753$ & 986,208 & $2,919,517$ \\
& & & & \\
Dynamic ( & & & & \\
$8 \%$ & & & & \\
$9 \%$ & $2,230,474$ & $1,621,935$ & $1,045,616$ & $2,697,116$ \\
$10 \%$ & $2,099,951$ & $1,643,770$ & $1,020,837$ & $2,503,541$ \\
$11 \%$ & $2,243,825$ & $1,762,712$ & 988,573 & $2,695,902$ \\
$12 \%$ & $2,263,690$ & $1,784,950$ & 968,086 & $2,815,685$ \\
All values in nominal dollars & $2,301,867$ & $1,777,479$ & 962,696 & $3,017,384$ \\
\hline & & & & \\
\hline
\end{tabular}


Table 7. Shortfall Probabilities of Dynamic Strategies Relative to other Asset Allocation Strategies

\begin{tabular}{|c|c|c|c|c|}
\hline \multirow{2}{*}{$\begin{array}{l}\text { Target Rate } \\
\text { of Return }\end{array}$} & \multicolumn{4}{|c|}{ Shortfall Probability of Dynamic Lifecycle Strategies } \\
\hline & Lifecycle & $100 \%$ Stocks & Balanced & $\begin{array}{l}\text { Target } \\
\text { Accumulation }\end{array}$ \\
\hline \multicolumn{5}{|c|}{ Dynamic ( $D L C_{20,20}$ ) } \\
\hline $8 \%$ & $18 \%$ & $63 \%$ & $8 \%$ & $25 \%$ \\
\hline $9 \%$ & $18 \%$ & $58 \%$ & $9 \%$ & $36 \%$ \\
\hline $10 \%$ & $19 \%$ & $51 \%$ & $10 \%$ & $48 \%$ \\
\hline $11 \%$ & $19 \%$ & $44 \%$ & $10 \%$ & $62 \%$ \\
\hline $12 \%$ & $20 \%$ & $37 \%$ & $11 \%$ & $73 \%$ \\
\hline \multicolumn{5}{|c|}{ Dynamic ( $D L C_{30,10}$ ) } \\
\hline $8 \%$ & $25 \%$ & $57 \%$ & $9 \%$ & $25 \%$ \\
\hline $9 \%$ & $26 \%$ & $51 \%$ & $10 \%$ & $35 \%$ \\
\hline $10 \%$ & $26 \%$ & $43 \%$ & $11 \%$ & $48 \%$ \\
\hline $11 \%$ & $27 \%$ & $36 \%$ & $12 \%$ & $60 \%$ \\
\hline $12 \%$ & $26 \%$ & $28 \%$ & $12 \%$ & $71 \%$ \\
\hline
\end{tabular}


Table 8. Average Shortfall of Dynamic Strategies Relative to other Asset Allocation Strategies

\begin{tabular}{|c|c|c|c|c|}
\hline \multirow[t]{2}{*}{$\begin{array}{l}\text { Target Rate } \\
\text { of Return }\end{array}$} & \multicolumn{4}{|c|}{ Average Shortfall of Dynamic Lifecycle Strategies } \\
\hline & Lifecycle & $100 \%$ Stocks & Balanced & $\begin{array}{l}\text { Target } \\
\text { Accumulation }\end{array}$ \\
\hline \multicolumn{5}{|c|}{ Dynamic ( $D L C_{20,20}$ ) } \\
\hline $8 \%$ & 29,259 & 699,151 & 5,030 & 87,329 \\
\hline $9 \%$ & 30,795 & 645,052 & 5,693 & 177,576 \\
\hline $10 \%$ & 34,462 & 582,815 & 6,110 & 335,465 \\
\hline $11 \%$ & 36,302 & 551,829 & 6,427 & 610,987 \\
\hline $12 \%$ & 40,151 & 479,332 & 6,667 & $1,033,334$ \\
\hline \multicolumn{5}{|c|}{ Dynamic $\left(D L C_{30,10}\right.$ ) } \\
\hline $8 \%$ & 42,982 & 392,565 & 5,873 & 90,599 \\
\hline $9 \%$ & 47,458 & 378,093 & 6,445 & 179,680 \\
\hline $10 \%$ & 50,273 & 343,890 & 6,907 & 340,492 \\
\hline $11 \%$ & 52,085 & 303,475 & 7,215 & 612,198 \\
\hline $12 \%$ & 55,808 & 250,991 & 7,147 & $1,027,964$ \\
\hline
\end{tabular}


Table 9. VaR and ETL Estimates of Dynamic Strategies for Different Target Returns

\begin{tabular}{|c|c|c|c|c|}
\hline \multirow{2}{*}{$\begin{array}{l}\text { Target } \\
\text { Return }\end{array}$} & \multicolumn{3}{|c|}{ VaR at Different Confidence Levels } & \multirow{2}{*}{$\begin{array}{l}\text { ETL at } 95 \% \text { Confidence } \\
\text { Level }\end{array}$} \\
\hline & $99 \%$ & $95 \%$ & $90 \%$ & \\
\hline \multicolumn{5}{|c|}{ Dynamic ( $D L C_{20,20}$ ) } \\
\hline $8 \%$ & 268,437 & 466,517 & 644,555 & 348,752 \\
\hline $9 \%$ & 273,801 & 458,214 & 615,008 & 348,786 \\
\hline $10 \%$ & 275,914 & 461,640 & 607,872 & 344,437 \\
\hline $11 \%$ & 261,970 & 450,583 & 603,010 & 344,610 \\
\hline $12 \%$ & 273,061 & 457,318 & 589,354 & 344,515 \\
\hline \multicolumn{5}{|c|}{ Dynamic ( $D L C_{30,10}$ ) } \\
\hline $8 \%$ & 268,431 & 459,189 & 630,391 & 345,246 \\
\hline $9 \%$ & 275,827 & 442,160 & 596,622 & 334,772 \\
\hline $10 \%$ & 274,968 & 444,468 & 599,673 & 340,901 \\
\hline $11 \%$ & 274,429 & 439,870 & 583,244 & 338,509 \\
\hline $12 \%$ & 267,946 & 447,535 & 592,663 & 338,154 \\
\hline All value & & & & \\
\hline
\end{tabular}

Another interesting point revealed by the sensitivity analysis is that as the target rate increases, the median outcome of the dynamic strategy continues to outperform that of the $100 \%$ stocks strategy by an even larger margin and vice versa. For example, by setting the target rate to $12 \%$ instead of $10 \%$, the margin of outperformance of $D L C_{20,20}$ over the $100 \%$ stock strategy increases by nearly $\$ 40,000$. The higher target also enables the dynamic strategies to close the gap with the $100 \%$ stock strategy in terms of mean and third quartile estimates. On the other hand, by setting a higher return target, the dominance of the first quartile outcome of the dynamic strategy over the corresponding outcome of the $100 \%$ stock strategy gets diminished considerably. In fact, $D L C_{30,10}$ actually produces a lower first quartile result than $100 \%$ stocks strategy when target rate for switching is set to $12 \%$. Similarly, the dominance of dynamic strategies over $100 \%$ stocks strategy for more inferior (below first quartile) outcomes is also adversely affected by raising the target rate for switching. 
The above findings are as would be expected - the higher one sets the target rate, the higher is the likelihood that the accumulation at any point would fall below the target thereby prompting the dynamic strategy to remain invested in equities. As a result, the behavior of the dynamic strategy would closely follow that of the $100 \%$ stocks strategy. The outcomes in the 'above median' range will get better, but outcomes in the 'below median' and 'below first quartile' range would become marginally poorer. On the other hand, if the target rate is set lower, there is a higher likelihood that the retirement account balance would cross the accumulation target at any point triggering the dynamic strategy to shift allocation towards bonds and cash. This, in turn, would cause the strategy to closely resemble a conventional lifecycle strategy

\section{Conclusion}

The evidence presented in this paper exposes the inherent weakness of traditional lifecycle investing for members of retirement plans. By blindly rolling over to conservative assets in the later part of the accumulation phase of retirement saving, lifecycle funds seem to be missing a trick. While switching out of volatile assets like stocks as the plan member nears retirement is generally accepted as sensible investment advice, traditional lifecycle funds appear to implement this strategy in a dogmatic manner that disregards the investors' wealth accumulation objectives.

As we have demonstrated, the mechanistic switching strategy from growth to conservative assets following any age-based rule of thumb is inferior to a dynamic strategy that considers the actual accumulation in the retirement account before switching assets. This paper proposes a specific dynamic asset allocation strategy where the switching of assets at any stage is based on cumulative investment performance of the portfolio relative to the investors' target at that stage. Unlike conventional lifecycle asset allocation rules where the switching of assets is preordained to be unidirectional, this dynamic strategy can switch assets in both directions: from aggressive to conservative and vice versa. Using the simple rule of almost stochastic dominance, we show that such a dynamic lifecycle strategy would be preferred to the conventional lifecycle strategy by most retirement plan members.

When comparing percentile outcomes in our trials, the only occasion when we find lifecycle strategies do better than the dynamic strategies is in outcomes below the $5^{\text {th }}$ to $10^{\text {th }}$ percentile range. However, the differences do not appear to be large enough to negate the appeal of dynamic strategies to the average investor in view of their overall dominance over lifecycle strategies. Even for these extremely adverse wealth outcomes in our trials, we find that the static balanced asset allocation strategy generally does better than the lifecycle strategy. Therefore an investor whose sole concern is improving the floor level of the extremely adverse wealth outcomes is likely to prefer investing in a balanced fund rather than a lifecycle fund. 
We have conducted a large number of trials in this paper to capture different possibilities about future asset class returns over the investment horizon of the retirement plan investor. According to our results, the chance of the dynamic strategy underperforming the lifecycle strategy at the end of such a long horizon is small (though not insignificant). Not only does the dynamic strategy produce superior terminal wealth outcomes compared to the lifecycle strategy in a vast majority (about 75 $80 \%$ ) of cases, it appears to have a fair chance of outperforming a $100 \%$ equity strategy. In fact, the dynamic lifecycle strategy $D L C_{30,10}$ in this paper which invests in an all equity portfolio for the first 30 years and then adjusts asset allocation on an annual basis, seems to have more than even chance of beating the strategy which invests in an all equity portfolio for the entire horizon.

It is hard to imagine that most people are so pessimistic or optimistic that they care only about the extreme outcomes. Decisions in life, including investment, are typically driven by the vast middle range of possibilities. It is precisely because of this reason that the dynamic strategy looks appealing in the context of our problem. Ignoring the extremities, the dynamic strategy invariably results in much higher wealth accumulation potential compared to the conventional lifecycle strategy. Remarkably, this is achieved while reducing downside risk compared to an all equity strategy as evidenced from the dominance of the dynamic strategy in the below median range of wealth outcomes.

In terms of practical considerations for implementation of the dynamic approach discussed in this paper, an important one is setting the target accumulation rate. If set too high, it is unlikely to be achieved and hence the investment strategy will remain $100 \%$ stocks for most of the accumulation period. If set too low, the overall strategy may be too conservative and in essence similar to the deterministic lifecycle strategy. There are also behavioural considerations. The dynamic strategy will switch back from conservative to growth assets in the final phases of the accumulation period if cumulative returns are below target. This is most likely to happen following poor returns in equities. It is likely that many unsophisticated investors, as will be typical participants in retirement plans, will be concerned about the prospect of increasing equity risk with recent losses still fresh in the mind. Hence, the strategy may be sensible from an investment perspective, particularly if there is a degree of mean reversion in returns, but difficult psychologically. An alternative might be an asymmetric dynamic approach that 'banks' excess gains, but does not increase risk when returns are below target.

Overall, it appears that dynamic lifecycle strategies that respond to achieved investment performance offer scope to improve on the deterministic strategies currently most common in use. We do not suggest that the specific dynamic allocation rule proposed in this paper is the optimal strategy for all, or even most, retirement investors. But we do think that our evidence points towards the general approach that practitioners should consider in designing lifecycle funds for retirement plans. 


\section{References}

Basu, A., and M. Drew. 2008. "Portfolio Size Effect in Retirement Accounts: What does it Imply for Lifecycle Asset Allocation Funds." Journal of Portfolio Management, Forthcoming.

Blake, D., A. Cairns, and K. Dowd. 2001. “Pensionmetrics: Stochastic Pension Plan Design and Value-atrisk during the Accumulation Phase." Insurance: Mathematics and Economics, vol. 29, 187-215.

Bodie Z. and J. Treussard. 2007. "Making Investment Choices as Simple as Possible, but Not Simpler." Financial Analysts Journal, vol. 63:3, 42-47.

Booth, P., and Y. Yakoubov. 2000. "Investment Policy for Defined-contribution Pension Scheme Members Close to Retirement: An Analysis of the 'Lifestyle" Concept'." North American Actuarial Journal, vol. 4, 1-19.

Byrne, A., D. Blake, A. Cairns, and K. Dowd. 2007. "Default Funds in UK Defined-Contribution Plans." Financial Analysts Journal, vol. 63, 42-51.

Cairns, A., D. Blake, and K. Dowd. 2006. "Stochastic Lifestyling: Optimal Dynamic Asset Allocation for Defined Contribution Pension Plans" Journal of Economic Dynamics and Control, vol. 30, 843-877.

Dimson, E., P. Marsh, and M. Staunton. 2002. Triumph of the Optimists: 101 Years of Global Investment Returns. Princeton, NJ: Princeton University Press.

Leshno, M., Levy, H., 2002. Preferred by “all” and preferred by “most" decision makers: Almost stochastic dominance. Management Science, vol. 48, 1074-1085.

Levy, H., 2006. Stochastic Dominance. Boston, MA: Kluwer Academic Publishers.

Munnell, A., and A. Sunden. 2006. “401(k) Plans Are Still Coming Up Short.” Issues in Brief No. 43, Centre for Retirement Research, Boston College, MA.

Poterba, J., and L. Summers. 1988. "Mean Reversion in Stock Prices: Evidence and Implications." Journal of Financial Economics, vol. 22, 27-59.

Samuelson, P. 1989. "The Judgment of Economic Science on Rational Portfolio Management: Timing and Long-horizon Effects." Journal of Portfolio Management, vol.16, 4-12.

Shiller, R. 2005. "Lifecycle Portfolios as Government Policy." The Economists' Voice, vol. 2, Article 14. Siegel, J. 2003. Stocks for the Long Run, The Definitive Guide to Financial Market Returns and Longterm Investment Strategies. New York: McGraw Hill 


\section{Notes}

\footnotetext{
${ }^{1}$ This is sometimes referred to as 'time diversification'. Samuelson (1989) shows that if returns are independently and identically distributed such long horizon effect cannot exist. While Samuelson's argument is mathematically sound, mean reversion in stock returns is a well documented empirical phenomenon. For example, Poterba and Summers (1988), provides evidence from the US market.
}

${ }^{2}$ Blake et al. (2001) test an asset switching strategy that incorporates performance feedback. The similarity between their threshold strategy and the dynamic lifecycle strategy proposed in this paper is that both resort to aggressive asset allocation if the portfolio underperforms the set benchmark (or lower threshold in their case) and vice versa. However, their strategy switches assets based on performance feedback right after the member joins the retirement plan while in this study the asset switching starts a few years before retirement which is akin to the switching principle adopted by most lifecycle funds in retirement plans. Moreover, they also set two distinct thresholds (upper and lower) to determine the direction and extent of asset switching while asset switching in this paper is governed by a single target return set by the investor.

${ }^{3}$ Cairns et al. (2006) investigate optimal dynamic asset allocation strategies for DC investors taking account of the investor's risk aversion and the assumed correlation between the member's salary and asset returns. Our paper makes a more direct comparison of the wealth outcomes of deterministic and dynamic lifecycle strategies.

${ }^{4}$ Munnell and Sunden (2006) suggest that the typical contribution rate for a $401(\mathrm{k})$ plan member is $9 \%$.

${ }^{5}$ Since the distribution of wealth outcomes get increasingly asymmetric over long horizons, the mean-variance framework is not useful in this situation. We also refrain from making any strong assumption on the utility function (like quadratic) of the retirement plan members.

${ }^{6}$ See Levy (2006) for a review of different applications of Stochastic Dominance.

${ }^{7}$ A rule under weaker condition called Second Degree Stochastic Dominance (SSD) is also applied to a large class of problems which works within the framework of risk aversion. Formally, given $U^{\prime}(W) \geq 0$ and $U^{\prime \prime}(W) \leq 0$, F is preferred to $\mathrm{G}$ under SSD criterion if and only if

\section{$\int_{0}^{\infty} F(W) d W \leq \int_{0}^{\infty} G(W) d W \quad \forall W$}

This implies that the area under $F$ has to be equal or less than the area under $G$ for the dynamic strategy for every $W$ to dominate the conventional strategy by the SSD rule.

${ }^{8}$ Take for instance the case where an investor face a choice between two uncertain prospects - a certain outcome $\mathrm{X}$ returning $\$ 1$ and an uncertain outcome $Y$ returning $\$ 100,000$ with probability of 0.99 and $\$ 0.9$ with a probability of 0.01 . Whilst it is practically inconceivable that any investor would not prefer F over G, under both FSD and SSD conditions $Y$ does not dominate $X$. The reason for this perverse result is that the stochastic dominance approach relates to all utility functions in a given class and therefore does not rule out extreme utility functions that provide higher expected utility under $\mathrm{X}$. 